\title{
Muon anomalous magnetic moment in the standard model with two Higgs doublets
}

\author{
Yue-Liang $\mathrm{Wu}$ and Yu-Feng Zhou \\ Institute of Theoretical Physics, Chinese Academy of Sciences, \\ Beijing 100080, China
}

\begin{abstract}
The muon anomalous magnetic moment is investigated in the standard model with two Higgs doublets (S2HDM) motivated from spontaneous $\mathrm{CP}$ violation. Thus all the effective Yukawa couplings become complex. As a consequence of the non-zero phase in the couplings, the one loop contribution from the neutral scalar bosons could be positive and negative relying on the $\mathrm{CP}$ phases. The interference between one and two loop diagrams can be constructive in a large parameter space of CP-phases. This will result in a significant contribution to muon anomalous magnetic moment even in the flavor conserving process with a heavy neutral scalar boson $\left(m_{h} \sim 200 \mathrm{GeV}\right)$ once the effective muon Yukawa coupling is large $\left(\left|\xi_{\mu}\right| \sim 50\right)$. In general, the one loop contributions from lepton flavor changing scalar interactions become more important. In particular, when all contributions are positive in a reasonable parameter space of CP phases, the recently reported 2.6 sigma experiment vs. theory deviation can be easily explained even for a heavy scalar boson with a relative small Yukawa coupling in the S2HDM.
\end{abstract}


Recently, the E821 Collaboration at BNL has reported their improved result on the measurement of muon anomalous magnetic moment $a_{\mu}=\left(g_{\mu}-2\right) / 2$ [1]. The difference between the measurement and the Standard Model (SM) prediction [2] is reported

$$
\Delta a_{\mu}=a_{\mu}^{e x p}-a_{\mu}^{S M}=426 \pm 165 \times 10^{-11},
$$

which shows a relative large deviation $(2.6 \sigma)$ from the SM calculation. At $95 \%$ confidence level, $\Delta a_{\mu}$ lie in the range

$$
113 \times 10^{-11} \leq \Delta a_{\mu} \leq 749 \times 10^{-11} .
$$

As muon is about 210 times heavier than electron, it is expected that the new physics effects on muon anomalous moment may be considerable. A large amount of works have been made on checking the new physics contributions to $\Delta a_{\mu}$ from various models. Among those, the contributions from supersymmetric particles and scalar bosons as well as extra dimensions seem to be the most attractive ones [3 -7]. In this paper, we shall make a systematic analysis in the standard model with two Higgs doublets (S2HDM) motivated from the study of origin and mechanism of $\mathrm{CP}$ violation [8 10$]$. In this kind of model, the Higgs sector of SM is simply extended by including an additional Higgs doublet with all the Yukawa couplings being real. After spontaneous symmetry breaking, the origin of both fermion masses and CP-violating phases can be attributed to the well known Higgs mechanism with a single CP-phase between two vacuum expectation values. Namely CP symmetry is broken spontaneously [11]. Thus all the effective Yukawa couplings in the physical basis become complex. The S2HDM has also been investigated from other considerations [12 17]. It will be seen that though S2HDM is the simplest extension of SM, it can provide a possible good explanation for the recently reported 2.6 sigma experiment vs. theory deviation of the muon anomalous magnetic moment.

There are several versions of 2HDM. To avoid flavor changing neutral current (FCNC) at tree level, some kind of discrete symmetries are often imposed on the Higgs potential, this results in two kinds of models which are usually called the 2HDM of type I and $\mathbb{I}[18$. Note that by imposing the discrete symmetry, the spontaneous CP violation is forbidden with two Higgs doublets and all the Yukawa couplings have to be real.

Contributions to muon $g-2$ from the 2HDM of type II have been discussed in Refs. [4]. It seems that the one loop contribution is not large enough to explain the data. Even for a large value of $\tan \beta \sim 50$, one still needs a very light mass of the scalar $M_{h} \sim 5 \mathrm{GeV}$. If both the scalar and pseudo-scalar are included, due to the cancelation between them, the situation will be even worse. One then needs to consider two loop contributions. It was found [5,6] that from the two-loop diagrams of Barr-Zee type [20], the contributions from pseudo-scalar is positive and could be larger than its negative one loop contributions. Thus the net effects for pseudo-scalar exchange become positive. Provided a sufficient large value of the coupling $\tan \beta$, its contributions can reach the experimental bound. By including the two loop diagrams, the pseudo-scalar mass must be below $80 \mathrm{GeV}$ even when $\tan \beta$ is large and around 50 [6]. To avoid the cancelation between scalar and pseudo-scalar exchange, the mass of the scalar boson has to be pushed to be very heavy ( typically greater than 500 $\mathrm{GeV})$.

An alternative way of suppressing the FCNC at tree level is to take the smallness of the off-diagonal elements in the Yukawa coupling matrices by considering approximate flavor or 
family symmetries [13, 16, 8 10]. It has been shown 8-10 that through the spontaneous CP violation, rich sources of $\mathrm{CP}$ violation including the KM-phase in SM can be induced from a single relative phase between the vacuum expectation values of the two Higgs doublets. In the S2HDM, as there are flavor changing scalar interactions for both neutral and charged scalar bosons, the bounds on the neutral Higgs mass can be released through the inclusion of the internal $\tau$ loop at one loop level [7]. Besides this, as it will be shown below that the complex and flavor dependent Yukawa couplings in S2HDM may completely change the interference between one and two loop diagram contributions. As a consequence, one and two loop contributions can be both positive and provide significant contributions to $\Delta a_{\mu}$.

In the S2HDM, after spontaneous symmetry breaking, the two Higgs fields are given by 810

$$
\phi_{1}=\left(\begin{array}{c}
\phi_{1}^{+} \\
\frac{1}{\sqrt{2}}\left(v \cos \beta e^{i \delta}+\phi_{1}^{0}\right)
\end{array}\right), \quad \phi_{2}=\left(\begin{array}{c}
\phi_{2}^{+} \\
\frac{1}{\sqrt{2}}\left(v \sin \beta+\phi_{2}^{0}\right)
\end{array}\right) .
$$

where the phase $\delta$ is the relative phase between the vacuum expectation values of the two Higgs doublets. This phase is the physical one as it can not be rotated away by a redefinition of the fermion phase. Thus in the physical basis (i.e., mass eigenstate basis), all the effective Yukawa couplings become complex, which leads to rich sources of CP violation. It is more natural to use another basis for Higgs fields through the recombination

$$
H_{1}=\phi_{1} \sin \beta e^{-i \delta}-\phi_{2} \cos \beta, \quad H_{2}=\phi_{2} \cos \beta e^{-i \delta}+\phi_{2} \sin \beta
$$

such that

$$
H_{1}=\left(\begin{array}{c}
H^{+} \\
\frac{1}{\sqrt{2}}(R+i I)
\end{array}\right), H_{2}=\left(\begin{array}{c}
0 \\
\frac{1}{\sqrt{2}}\left(v+H^{0}\right)
\end{array}\right)
$$

where $H^{+}$is the charged scalar and $R, I$ and $H^{0}$ are the three neutral scalar bosons. Here the neutral Higgs boson $H^{0}$ plays the role as the one in SM with one Higgs doublet. The additional two neutral scalar bosons $R$ and $I$ will lead to new interactions beyond the SM.

The Yukawa interactions between scalar bosons and leptons in the S2HDM can be written as the sum of two parts $\mathcal{L}_{1}$ and $\mathcal{L}_{2}$, where $\mathcal{L}_{1}$ contains only flavor conserving interactions and $\mathcal{L}_{2}$ contains flavor changing ones, i.e.

$$
\mathcal{L}_{Y}=\left(\sqrt{2} G_{F}\right)^{1 / 2}\left(\mathcal{L}_{1}+\mathcal{L}_{2}\right)
$$

with

$$
\begin{aligned}
& \mathcal{L}_{1}=\sqrt{2} H^{+} \sum_{i}^{3} \xi_{l_{i}} m_{l_{i}} \bar{\nu}_{L}^{i} l_{R}^{i}+H^{0} \sum_{i}^{3} m_{l_{i}} \bar{l}_{L}^{i} l_{R}^{i}+(R+i I) \sum_{i}^{3} \xi_{l_{i}} m_{l_{i}} \bar{l}_{L}^{i} l_{R}^{i}+h . c, \\
& \mathcal{L}_{2}=(R+i I) \sum_{i \neq j}^{3} \xi_{l_{i} l_{j}} \bar{l}_{L}^{i} l_{R}^{j}+h . c
\end{aligned}
$$

In the above expression, $\xi_{l_{i}}$ and $\xi_{l_{i} l_{j}}$ stand for the flavor conserving and flavor changing Yukawa couplings which are in general all complex numbers. For simplicity, we will neglect, in the following considerations, the mixing among the three neutral scalar bosons, but their 
masses are considered to be largely split and the mass eigenstates are denoted by $h$ and $A$ respectively.

The phase effects of the Yukawa coupling has been discussed in the literatures 8 10,21,23. The complex couplings may lead to sizable CP violation in charged and neutral Higgs boson exchanging processes in quark sector, such as $b \rightarrow s \gamma$ 9, 23, 22, $K^{0}-\bar{K}^{0}$, $B^{0}-\bar{B}^{0}, B_{s}^{0}-\bar{B}_{s}^{0}$ and $D^{0}-\bar{D}^{0}$ mixings [24], etc. In the lepton sector, it may result in large electric dipole moment of leptons [21, 10, 26].

In the case of effective real Yukawa couplings for neutral scalar bosons, $h$ is purely a scalar while $A$ is a pseudo-scalar. The contributions to muon anomalous moment from these two particles always have different signs at both one loop and two loop level. Namely, for scalar $h$-exchange, the contribution is positive from one loop and negative from two loop. But for pseudo-scalar $A$-exchange, the one loop contribution is always negative and the two loop contribution is positive. As the experimental data indicate that the deviation of $g-2$ from SM is positive. The large two loop contribution may lead to the conclusion that the $A$-exchange must be dominant and the $h$-exchange must be made to be negligible small.

However, in the general case, the situation can be quite different. As all the couplings are complex in S2HDM, the Yukawa couplings for neutral scalar bosons $h$ and $A$ contain both scalar and pseudo-scalar type fermion interactions. Further more, the couplings are all flavor dependent, which is far away from the 2HDM of type I and II, where the couplings are given by a simple function of $\tan \beta$. The fact that one loop and two loop contributions depend on different complex Yukawa couplings provides an alternative possibility that they may not always cancel each other. In a large parameter space, the two kind of contributions may be constructive and result in a large value of muon $g-2$

The above discussion is also suitable for SUSY model [5, 6] as the Higgs sector of it is exactly like the 2HDM of type II. Recently, the contribution from two loop diagrams with charged Higgs in SUSY is also discussed [27]. However, In the case of SUSY, the main contribution may arise from the chargino-sneutrino and neutralino-slepton couplings at one loops [28].

The one loop flavor conserving contributions from the scalar and pseudo-scalar have been investigated in Refs. [30]. In S2HDM, the results are given by

$$
\begin{aligned}
& \Delta a_{\mu}^{h}=\frac{g^{2}}{32 \pi^{2}} \frac{m_{\mu}^{4}}{m_{W}^{2} m_{h}^{2}}\left[\left(\operatorname{Re} \xi_{\mu}\right)^{2}\left(\ln \frac{m_{h}^{2}}{m_{\mu}^{2}}-\frac{7}{6}\right)-\left(\operatorname{Im} \xi_{\mu}\right)^{2}\left(\ln \frac{m_{h}^{2}}{m_{\mu}^{2}}-\frac{11}{6}\right)\right] \\
& \Delta a_{\mu}^{A}=\frac{g^{2}}{32 \pi^{2}} \frac{m_{\mu}^{4}}{m_{W}^{2} m_{A}^{2}}\left[\left(\operatorname{Im} \xi_{\mu}\right)^{2}\left(\ln \frac{m_{A}^{2}}{m_{\mu}^{2}}-\frac{7}{6}\right)-\left(\operatorname{Re} \xi_{\mu}\right)^{2}\left(\ln \frac{m_{A}^{2}}{m_{\mu}^{2}}-\frac{11}{6}\right)\right],
\end{aligned}
$$

where $g$ is the weak gauge coupling constant. In the limit of $m_{\mu}^{2} / m_{h}^{2} \ll 1$, the one loop results can be approximately written as

$$
\Delta a_{\mu}^{h(A)}= \pm \frac{g^{2}}{32 \pi^{2}} \frac{m_{\mu}^{4}}{m_{W}^{2} m_{h}^{2}} \ln \left(\frac{m_{h}^{2}}{m_{\mu}^{2}}\right)\left|\xi_{\mu}\right|^{2} \cos 2 \delta_{\mu}
$$

where $\delta_{\mu}$ is the phase of $\xi_{\mu}$ with $\xi_{\mu}=\left|\xi_{\mu}\right| e^{i \delta_{\mu}}$. The one loop flavor conserving contribution to $\Delta a_{\mu}$ is plotted in Fig.2 2a. It can be seen that its contributions decrease rapidly with $m_{h}$ increasing, which means that the one loop diagram cannot give large contributions to $\Delta a_{\mu}$ except for a very small value of $m_{h} \sim 10 \mathrm{GeV}$ or a very large value of $\left|\xi_{\mu}\right| \sim 70$. Note that 
in the S2HDM, the contributions from $h$ and $A$ can be negative and positive depending on the sign of $\cos 2 \delta_{\mu}$, which is completely different from the other type of $2 \mathrm{HDM}$, such as type I and type II, where the $h(A)$ loop diagrams always give positive (negative) contributions.

In the case of one loop flavor changing processes ( see Fig.1 b), the loops with internal $\tau$-lepton play an important role as it is much heavier than the $\mu$-lepton. This then leads to an enhancement factor of the order $m_{\tau}^{2} / m_{\mu}^{2} \sim \mathcal{O}\left(10^{2}\right)$ relative to the flavor conserving one. In S2HDM, the contributions from $h(A)$ exchange are given by

$$
\Delta a_{\mu}^{h(A)}= \pm \frac{g^{2}}{32 \pi^{2}} \frac{m_{\mu}^{2} m_{\tau}^{2}}{m_{W}^{2} m_{h(A)}^{2}}\left(\ln \frac{m_{h(A)}^{2}}{m_{\tau}^{2}}-\frac{3}{2}\right)\left|\xi_{\mu \tau}\right|^{2} \cos 2 \delta_{\mu \tau}
$$

In obtaining the above expression, we have taken $\xi_{\mu \tau}=\xi_{\tau \mu}$ for simplicity. The flavor changing contributions to $\Delta a_{\mu}$ are presented in Fig.2[ $\mathrm{b} * *$ with different values of $\left|\xi_{\mu \tau}\right|$. The figure shows that the contributions to $\Delta a_{\mu}$ may be considerable large when $\left|\xi_{\mu \tau}\right|$ is large. For $\left|\xi_{\mu \tau}\right|=30 \sim 50$, the recently reported 2.6 sigma experiment vs. theory deviation can be easily explained even for a heavy scalar boson $m_{h}>100 \mathrm{GeV}$. So far, there are no strict constraints on the values of the coupling $\left|\xi_{\mu \tau}\right|$. Studies on the rare decays $\tau \rightarrow \mu(e) \gamma$ and $\tau \rightarrow 3 \mu(e)$ and the electric dipole moment of $\tau$ will be useful to provide an interesting constraint on the parameter. However, as the relevant experimental data at present are primitive and such processes often contain more couplings such as $\xi_{\tau}$ and $\xi_{\tau e}$, the resulting constraints can not be clearly obtained and they are not yet be very strong. To obtain the upper bounds on $\xi_{\tau \mu}$, further studies are needed.

Now let us discuss the two loop effects on $\Delta a_{\mu}$. Naively speaking, relative to one loop diagram, the two loop diagram will receive an additional suppression factor of $\alpha / 4 \pi \sim \mathcal{O}\left(10^{-3}\right)$ and is in general small. However, there exists an special kind of two loop diagrams which can give significant contributions through the large Yukawa couplings between Higgs bosons and heavy fermions. These processes which are often referred as Barr-Zee mechanism are shown in Fig.(1.) $c$ and $d$. In this kind of diagrams, the Yukawa coupling will contribute a enhance factor of $m_{f} / m_{\mu}$. if $f$ is heavy quark such $t$ - quark, it can reach the order of $\mathcal{O}\left(10^{3}\right)$. Thus the two loop contribution could be sizeable. The large Yukawa couplings of order $\mathcal{O}(50)$ is need for explain the mass ratio between top and bottom quark in GUT [29]. Note that arbitrary large Yukawa couplings may lead the theory to be non-perturbative. This requires that the couplings have to be less than $1 /\left(\sqrt{2}\left(\sqrt{2} G_{f}\right)^{1 / 2} m_{f}\right)$, where $G_{f}$ is the Fermi constant. For example, for $t(b)$-quark, it is less than 1(44). In the virtual loop corrections, as there are additional loop suppression factor of $(4 \pi)^{-2}$. The constraints may be weak. Further more, the loop integrals always decrease with Higgs mass growing, and the Higgs loop contribution will decouple at large Higgs mass limit.

For the case that the fermion loop is dominated by top quark, the Barr-Zee type two loop diagrams may lead to a sizable $\Delta a_{\mu}$. In S2HDM, their contributions are given by

$$
\begin{aligned}
\Delta a_{\mu}^{h} & =\frac{N_{c} q_{t}^{2} \alpha^{2}}{4 \pi^{2} \sin ^{2} \theta_{W}} \frac{m_{\mu}^{2}}{m_{W}^{2}}\left[\operatorname{Im} \xi_{t} \operatorname{Im} \xi_{\mu} f\left(\frac{m_{t}^{2}}{m_{h}^{2}}\right)-\operatorname{Re} \xi_{t} \operatorname{Re} \xi_{\mu} g\left(\frac{m_{t}^{2}}{m_{h}^{2}}\right)\right] \\
\Delta a_{\mu}^{A} & =\frac{N_{c} q_{t}^{2} \alpha^{2}}{4 \pi^{2} \sin ^{2} \theta_{W}} \frac{m_{\mu}^{2}}{m_{W}^{2}}\left[\operatorname{Re} \xi_{t} \operatorname{Re} \xi_{\mu} f\left(\frac{m_{t}^{2}}{m_{A}^{2}}\right)-\operatorname{Im} \xi_{t} \operatorname{Im} \xi_{\mu} g\left(\frac{m_{t}^{2}}{m_{A}^{2}}\right)\right]
\end{aligned}
$$


where $N_{c}=3$ is the color number and $q_{t}=2 / 3$ is the charge of top quark. $\alpha=1 / 137$ and $\sin ^{2} \theta_{W}=0.23$ are the fine structure constant and weak mixing angle respectively. $f(z)$ and $g(z)$ are two integral functions which have the following form [20]

$$
\begin{aligned}
& f(z)=\frac{1}{2} z \int_{0}^{1} d x \frac{1-2 x(1-x)}{x(1-x)-z} \ln \frac{x(1-x)}{z} \\
& g(z)=\frac{1}{2} z \int_{0}^{1} d x \frac{1}{x(1-x)-z} \ln \frac{x(1-x)}{z}
\end{aligned}
$$

for large $z, f(z) \sim 1 / 3 \ln z+13 / 8, g(z) \sim 1 / 2 \ln z+1$ and $f(1) \sim 1 / 2, g(1) \sim 1$. The pure two loop contributions are plotted in Fig.2 $\mathrm{b}$. Unlike in the one loop case, where $\Delta a_{\mu}$ decreases rapidly with growing $m_{h}$, the two loop contributions decrease relative slowly and their signs depend on the value of $\delta_{\mu \tau}$. Therefore for a very large value of $m_{h}$, the two loop effects become dominant. Another difference is that in the one loop case the new physics contributions only depend on $\xi_{\mu}$, while the two loop contributions depend on two couplings $\xi_{\mu}$ and $\xi_{t}$, if the fermion loops for $b$ or $\tau$ are included [5.6], it will depend on more parameters. As the two couplings are complex numbers, the interference between one and two loop diagrams may not always be destructive. There exists a large parameter space in which the one and two loop contributions are all positive. Thus they can result in a large contribution to $\Delta a_{\mu}$. The constraint of $\left|\xi_{t}\right|$ has been studied in $B^{0}-\bar{B}^{0}$ mixing and $b \rightarrow s \gamma$ as well as the neutron electric dipole moment [24,9,10,21,25], the typical absolute value for $\left|\xi_{t}\right|$ is of the order $\mathcal{O}(1)$. Taking $\left|\xi_{t}\right|=1$ and $\delta_{t}=0$ as an example, the sum of one and two loop contributions from $h$-scalar exchange reads

$$
\Delta a_{\mu}=\frac{\alpha\left|\xi_{\mu}\right|}{\sin ^{2} \theta_{W}} \frac{m_{\mu}^{2}}{m_{W}^{2}}\left[\frac{\left|\xi_{\mu}\right|}{8 \pi} \frac{m_{\mu}^{2}}{m_{h}^{2}} \ln \left(\frac{m_{h}^{2}}{m_{\mu}^{2}}\right) \cos 2 \delta_{\mu}-\frac{\alpha}{3 \pi^{2}} g\left(\frac{m_{t}^{2}}{m_{h}^{2}}\right) \cos \delta_{\mu}\right] .
$$

It is clear that if $\delta_{\mu}$ lies in the range $3 \pi / 4 \leq \delta_{\mu} \leq 5 \pi / 4$, the one and two loop contributions will be constructive. The detailed numerical results for two different values of $\delta_{t}$ are plotted in Fig. 3 and Fig. 4 , where $\delta_{\mu}$ runs in a wide range. It can be seen that for large values of $\delta_{\mu} \sim \pi$, the contributions from $h$-scalar exchange can reach the experimental lower bound even for a heavy scalar with $m_{h} \sim 200 \mathrm{GeV}$. This is quite different from the existed 2HDM calculations in the literature, where the allowed range for the scalar boson $A$ must be less than $100 \mathrm{GeV}$, and the scalar boson $h$ must be much heavier than $A$, so that its negative contributions are negligible.

In a large parameter space, it is also possible that contributions from one loop flavor conserving and one loop flavor changing as well as two loop diagrams are all positive. In this case, the current data can be easily explained even with a relative small value of $\left|\xi_{\mu \tau}\right|$. The results for such a situation are plotted in Fig.5. It is seen that in this case a reasonable value of $\left|\xi_{\mu \tau}\right| \sim 10$ is large enough to reach the experiment bound.

In conclusion, we have investigated all possible new contributions to the muon anomalous magnetic moment from scalar interactions in S2HDM. Though S2HDM is regarded as the simplest extension of SM, it can provide a possible good explanation on the recently reported 2.6 sigma experiment vs. theory deviation. In particular, the important effects of the CP phases appearing in the Yukawa couplings have been studied. Unlike the 2HDM of type I and II, the two loop $h$ - scalar contribution to $\Delta a_{\mu}$ could be positive. The interference between 
one and two loop contributions can be constructive and result in a considerable contribution to $\Delta a_{\mu}$, so as to coincide with the recent E821 experiment data. In the case of constructive interference, the experimental data $\Delta a_{\mu}$ can be understood even for a heavy scalar boson $h$ with mass $m_{h} \sim 200 \mathrm{GeV}$, once the Yukawa coupling $\left|\xi_{\mu}\right|$ is large, $\left|\xi_{\mu}\right| \sim 50$. In general, contributions from one loop flavor changing diagrams are likely to be the dominant one in a large parameter space. Especially, in the case that contributions from all diagrams, namely, the one loop flavor conserving and one loop flavor changing as well as two loop diagrams in S2HDM with a reasonable parameter space, are all positive, one can easily explain the current experimental data of the excess of the muon anomalous magnetic moment.

\section{ACKNOWLEDGMENTS}

This work was supported in part by the NSF of China under the grant No. 19625514 as well as Chinese Academy of Sciences. 


\section{REFERENCES}

[1] H. N. Brown, et.al, Phys. Rev. Lett. 86 ,2 (2001)227.

[2] S. J. Brodsky and J. D. Sullivan, Phys. Rev. 156, 1644 (1967), T. Burnett and M. J. Levine, Phys. Lett. 24B, 467 (1967), M. Gourdin and E. de Rafael , Nucl. Phys. B10, 667 (1969), R. Jackiw and S. Weinberg, Phys. Rev. D5, 2473 (1972), K. Fujikawa, B. W. Lee, and A. I. Sanda, Phys. Rev. D6, 2923 (1972), I. Bars and M. Yoshimura, Phys. Rev. D6, 374 (1972), G. Altarelli, N. Cabibbo, and L. Maiani, Phys. Lett. B40, 415 (1972), W. A. Bardeen, R. Gastmans, and B. E. Lautrup, Nucl. Phys. B46, 315(1972), A. Czarnecki, B. Krause and W. Marciano, Phys. Rev. D52, R2619 (1995), S. Peris, M. Perrottet, and E. de Rafael, Phys. Lett. B355, 523 (1995), A. Czarnecki, B. Krause and W. Marciano, Phys. Rev. Lett. 76 ,3267 (1996), G. Degrassi and G. F. Giudice, Phys. Rev. D58, 053007 (1998), M. Davier and A. Höcker, Phys. Lett. B435, 427 (1998), M. Davier, Nucl. Phys. B (Proc. Suppl.) 76, 327 (1999).

[3] U. Chattopadhyay, D. K. Ghosh, S. Roy, hep-ph/0006049; L. Everett, G. L. Kane, S. Rigolin, L. T. Wang, hep-ph/0102145; J. L. Feng , K. T. Matchev, hep-ph/0102146; U. Chattopadhyay, P. Nath, hep-ph/0102157 ; S. Komine, T. Moroi, M. Yamaguchi, hep-ph/0102204; J. Hisano, K. Tobe, hep-ph/0102315; J. E. Kim, B. Kyae, H. M. Lee, hep-ph/0103054; S. P. Martin, J. D. Wells, hep-ph/0103067; H. Baer, C. Balazs, J. Ferrandis, X. Tata, hep-ph/0103280; G. C. McLaughlin, J. N. Ng, hep-ph/0008209; S. C. Park, H. S. Song, hep-ph/0103072; C. S. Kim, J. D. Kim, J. Song, hep-ph/0103127; K. Agashe, N.G. Deshpande, G.-H. Wu, hep-ph/0103235; K. Choi, et. al, hep-ph/0103048; E. Ma and M. Raidal, hep-ph/0102255; R. Arnowitt, B. Dutta, B. Hu and Y. Santoso, hep-ph/0102344; V.Barger, et. al, hep-ph/0101106 R. Casadio, A. Gruppuso and G. Venturi, hep-th/0010065;

[4] A. Dedes and H. E. Haber, hep-ph/0102297; M. Krawczyk, hep-ph/0103223.

[5] D. Chang, W. F. Chang, C. H. Chou and W. Y. Keung, hep-ph/0009292;

[6] K. Cheung, C. H. Chou and C. W. Kong, hep-ph/0103183.

[7] S. Nie and M. Sher, Phys. Rev. D58, 097701 (1998);

R. A. Diaz, R. Martinez and J. A. Rodriguez, hep-ph/0010339;

S.K. Kang and K. Y. Lee, hep-ph/0103064.

[8] Y.L. Wu and L. Wolfenstein, Phys. Rev. Lett. 73, 1762 (1994).

[9] L. Wolfenstein and Y.L. Wu, Phys. Rev. Lett., 73, 2809(1994).

[10] For more detailed analyses, see: Y.L. Wu, Carnegie-Mellon Report, hep-ph/9404241, 1994 (unpublished); A Model for the Origin and Mechanisms of CP Violation, in: Proceedings at 5th Conference on the Intersections of Particle and Nuclear Physics, St. Petersburg, FL, 31 May- 6 Jun 1994, pp338, edited by S.J. Seestrom (AIP, New York, 1995).

[11] T. D. Lee, Phys, Rep.9, 143-158 and 176-177 (1974).

[12] T.P.Cheng and M.Sher, Phys.Rev.D35,3484(1987).

[13] A. Antaramian, L.J. Hall and A. Rasin, Phys. Rev. Lett. 69, 1871 (1992).

[14] W.S. Hou, Phys. Lett. B296, 179 (1992);

[15] D. Chang, W.S. Hou and W.Y. Keung, Phys. Rev. D48, 217 (1993).

[16] L.J. Hall and S. Weinberg, Phys. Rev. D48, 979 (1993).

[17] D. Atwood, L. Reina, and A. Soni, Phys. Rev. D55, 3156 (1997). 
[18] J. F. Gunion, H. E. Haber, G. Kane and S. Dawson, The Higgs Hunter's Guide, Addison-Wesley (1990).

[19] M. Krawczyk and J. Zochowski, Phys. Rev. D55, 6968 (1997).

[20] S. M. Barr and A. Zee, Phys. Rev. Lett. 65 ,21 (1990); S.Weinberg. Phys. Rev. Lett. 63,2333 (1989).

[21] D. B. Chao, K. Cheung, and W. Y. Keung, Phys. Rev. D59, 115006 (1999).

[22] K. T. Mahanthappa and S. Oh,Phys. Rev. D62, 015012(2000)

[23] Y.L. Wu, Chin. Phys. Lett. 16 339,1999, hep-ph/9805439.

[24] Y.L. Wu and Y.F. Zhou Phys.Rev. D61, 096001(2000).

[25] Y. L. Wu and Y. F. Zhou, hep-ph/0102310.

[26] E. O. Iltan, hep-ph/0101017.

[27] C.H. Chen and C. Q. Geng, Phys.Lett. B511, 77 (2001).

[28] J.Lopez, D.V. Nanopoulos and X.Wang, Phys. Rev. D49,366 (1991);

[29] M. Olechowski and S. Pokorski, Phys. Lett. B214, 393 (1988); B. Anantharayan and G. Lazarides and Q. Shafi, Phys. Rev. D44, 1613 (1991); G. Anderson, S. Dimopoulos, L.J. Hall and S. Raby, Phys. Rev. D47, 3702 (1993); L.J. Hall, R. Rattazzi and U. Sarid, Phys. Rev. D50, 7048 (1994); M. Carena, M. Olechowski, S. Pokorski and C.E.M Wagner, Nucl. Phys. B426, 269 (1994); P. Langacker and N. Polonsky, Phys. Rev. D49, 1454 (1994). U. Chattopadhyay and P. Nath, Phys. Rev. D53,1648 (1996); T. Moroi, Phys. Rev. D53,6565 (1996); M. Carena, G. F. Giudice, and C. E. M. Wagner, Phys. Lett. B390,234 (1997); G.C. Cho, K. Hagiwara and M. Hayakawa, Phys. Lett.B478,231 (2000).

[30] J. R. Primack and H.R. Quinn, Phys. Rev. D6, 3171 (1972); W. A. Bardeen, R. Gastmans and B. Lautrup, Nucl. Phys. B 46, 319 (1972); J. P. Leveille, Nucl. Phys. B 137, 63 (1978); H. E. Haber, G. L. Kane and T. Sterling, Nucl. Phys. B 161, 493 (1979); E. D. Carlson, S.L. Glashow and U. Sarid, Nucl. Phys. B 309, 597 (1988); 


\section{FIGURES}

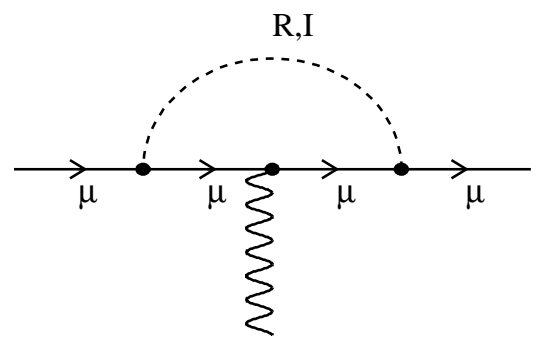

(a)

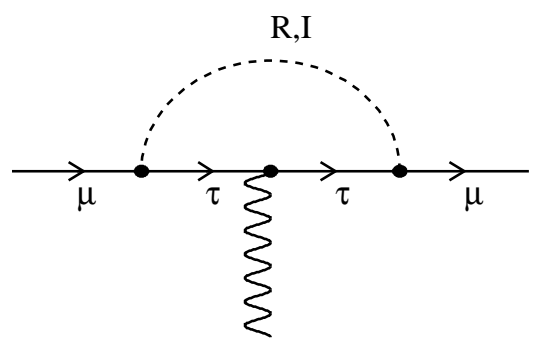

(b)
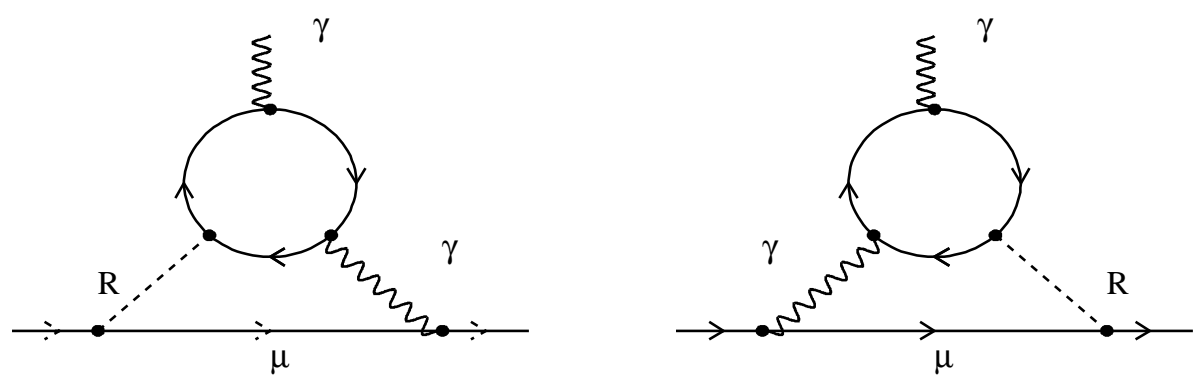

FIG. 1. Feynman diagrams contributing to $\Delta a_{\mu}$ in S2HDM.

(a) one loop flavor conserving.

(b) one loop flavor changing.

(c) and d) two loop diagrams of Barr-Zee type 

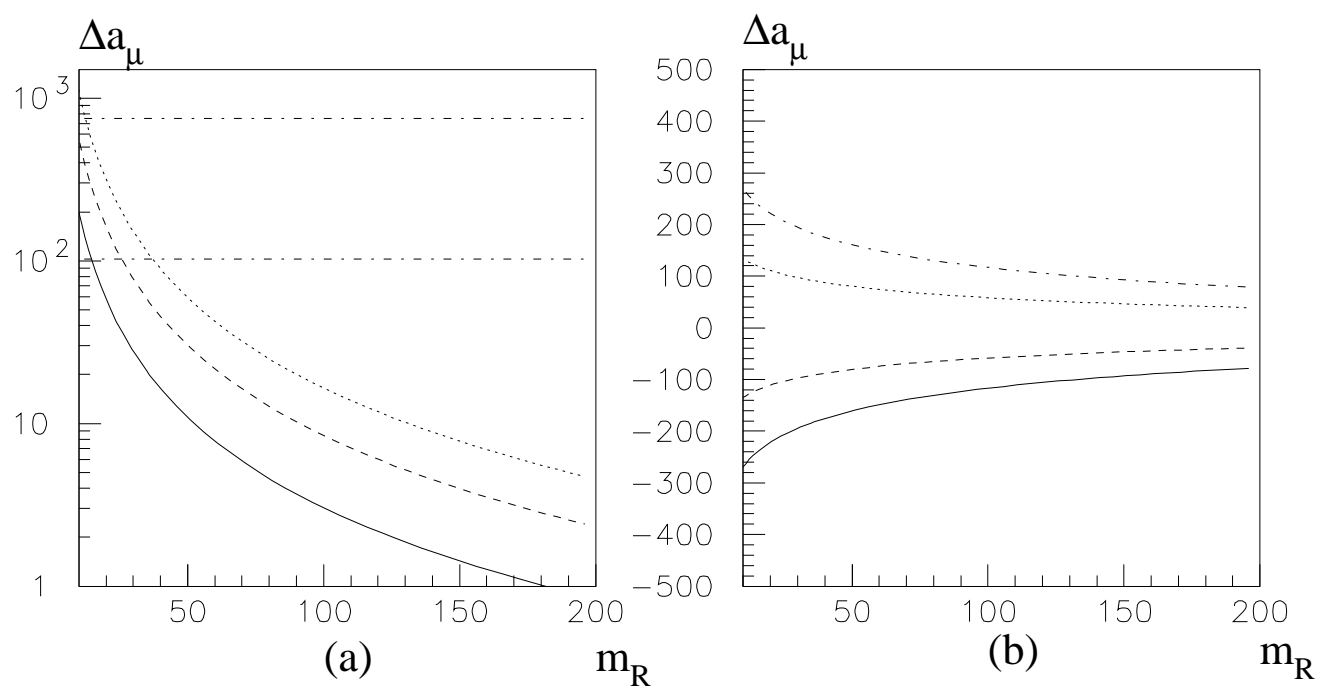

FIG. 2.

a) Single one loop flavor conserving contribution to $\Delta a_{\mu}$ as function of $m_{h}$. The solid, dashed and dotted cures correspond to $\left|\xi_{\mu}\right|=30,50,70$ respectively. The two dot-dashed horizontal lines present the current experimental bound at $95 \%$ confidence level

b)Single two loop contribution with $\left|\xi_{\mu}\right|$ being fixed at 50 and $\delta_{\mu}$ varies from $0, \pi / 3,2 \pi / 3$ to $\pi$ ( from down to up). 

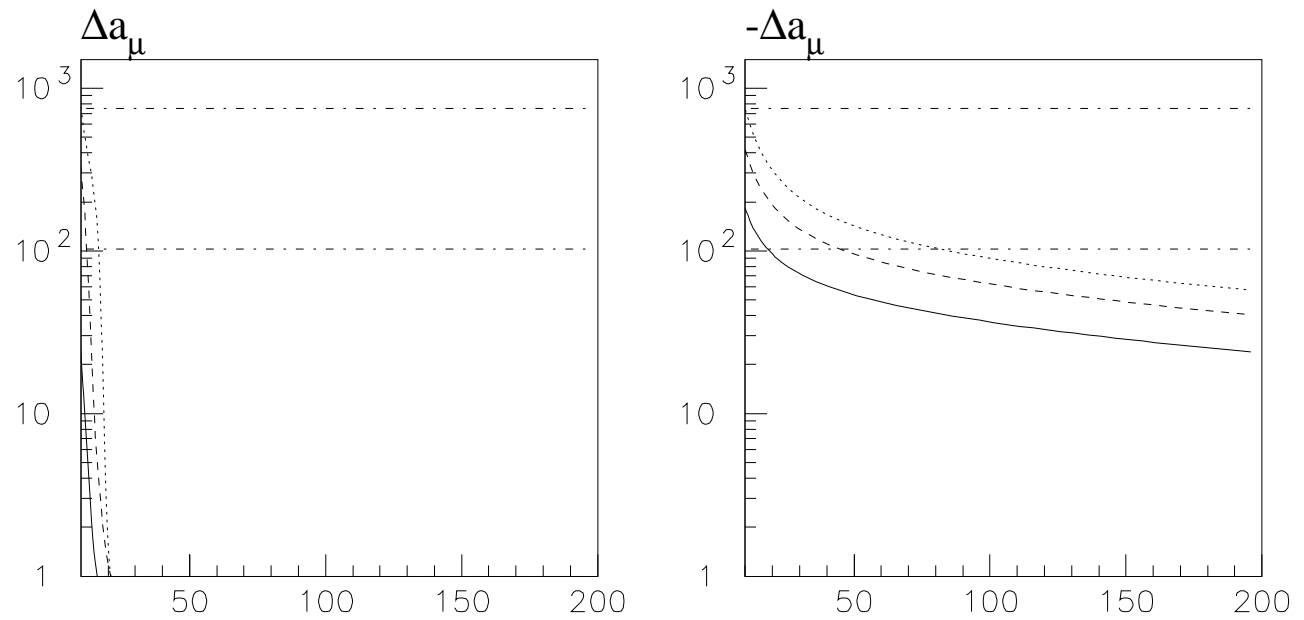

(a)

$\mathrm{m}_{\mathrm{R}}$

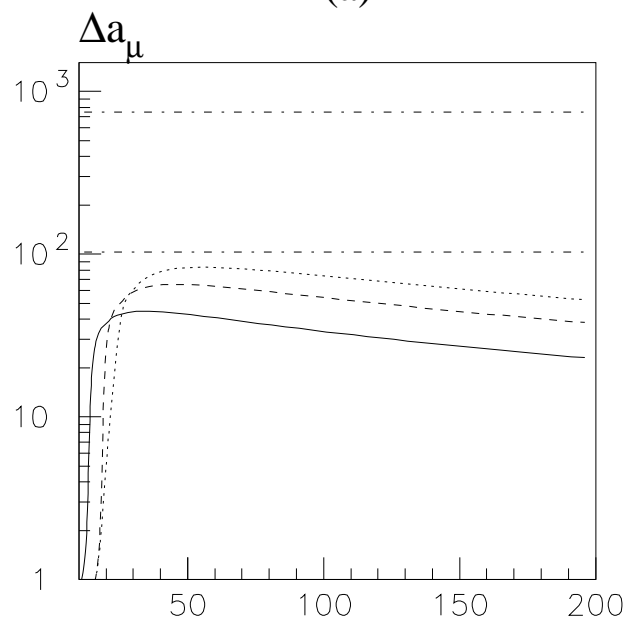

(b)

$\mathrm{m}_{\mathrm{R}}$

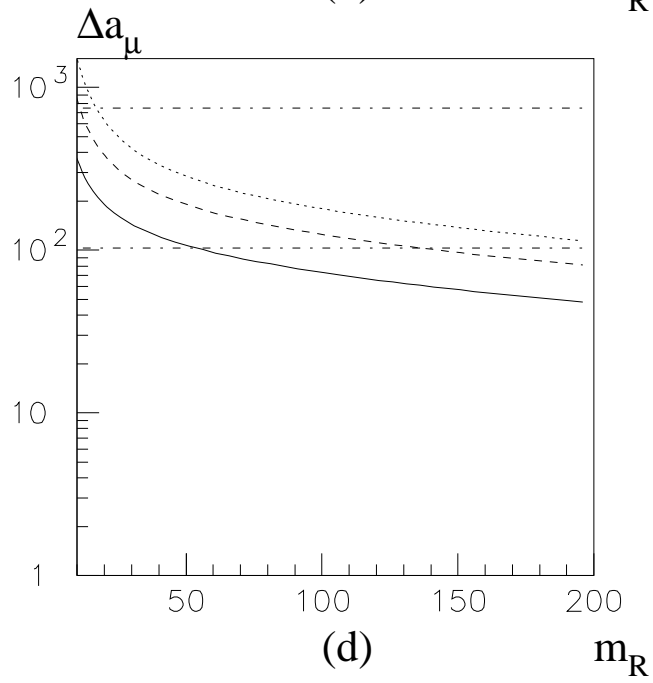

FIG. 3. The sum of the one loop flavor conserving and the two loop contribution to $\Delta a_{\mu}$ as function of $m_{h}$. for $\left|\xi_{t}\right|=1$ and $\delta_{t}=0$.

(a) The solid, dashed and dotted cures correspond to $\left|\xi_{\mu}\right|=30,50,70$ respectively, with $\delta_{\mu}=0$.

(b) The same as (a) with $\delta_{\mu}=\pi / 3$. Note that all the contributios are negative.

(c) The same as (a) with $\delta_{\mu}=2 \pi / 3$.

(d) The same as (a) with $\delta_{\mu}=\pi$. 

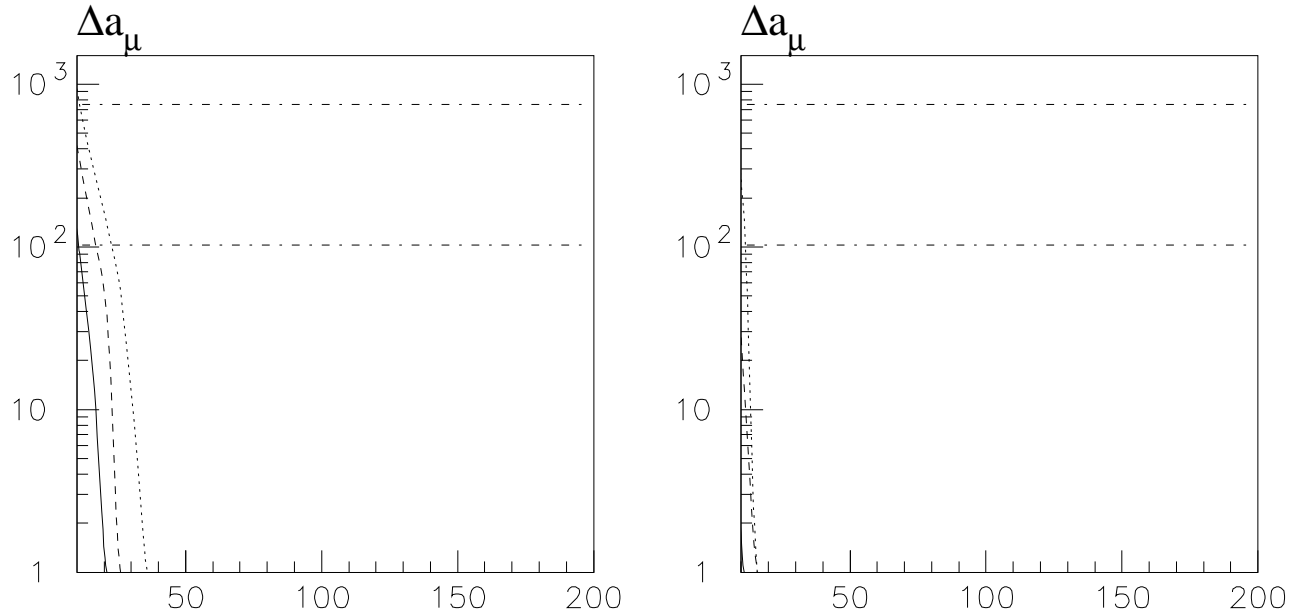

(a)
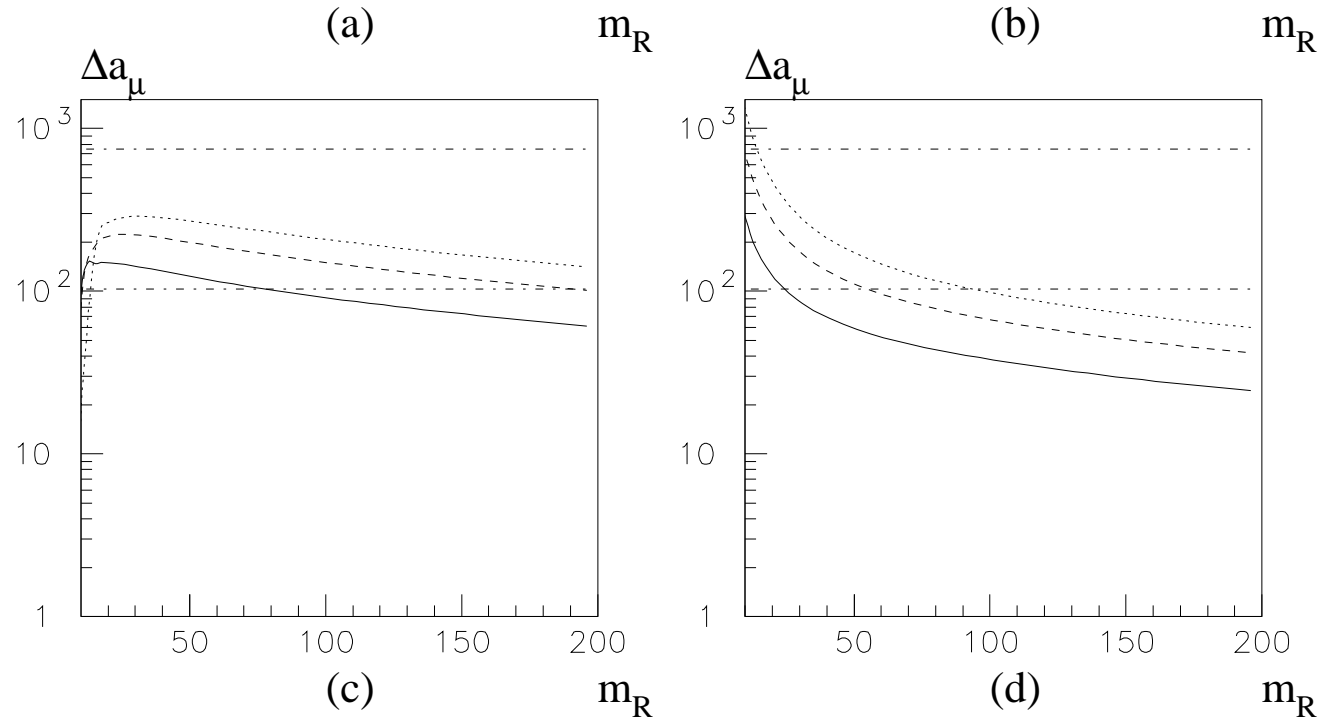

(c)

$\mathrm{m}_{\mathrm{R}}$

(d)

$\mathrm{m}_{\mathrm{R}}$

FIG. 4. The same as Fig. 3 for $\delta_{t}=\pi / 3$ 


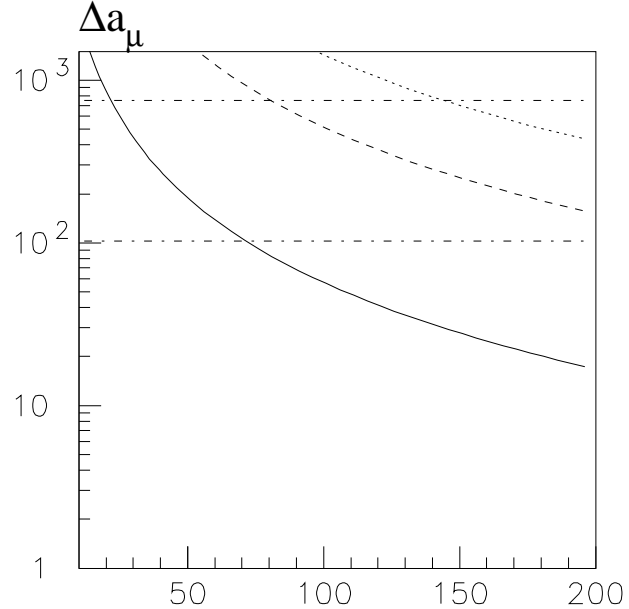

(a)

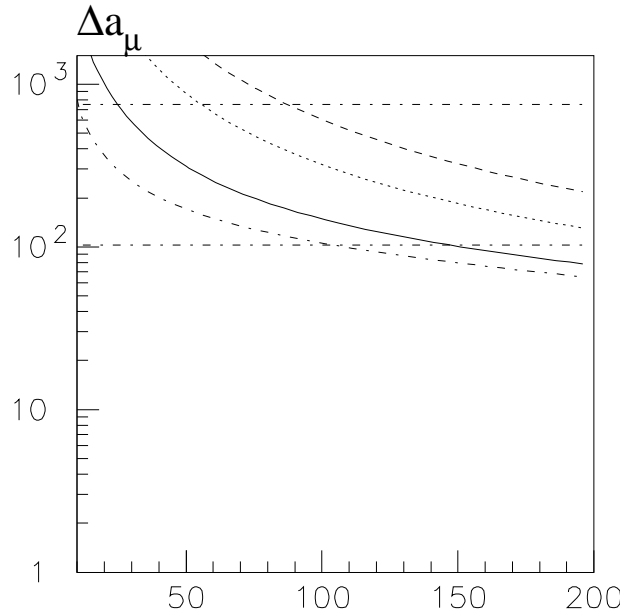

(b)
$\mathrm{m}_{\mathrm{R}}$

FIG. 5.

(a) The single flavor changing contributon, the solid, dashed and dotted curves corresponds to $\left|\xi_{\mu \tau}\right|=10,30,50$, with $\delta_{\mu \tau}=0$.

(b)The sum of one loop flavor conserving, one loop flavor changing and the two loop contributions with $\left|\xi_{t}\right|=1, \delta_{t}=\pi / 3$ and $\left|\xi_{\mu}\right|=30, \delta_{\mu}=2 \pi / 3$. The dot-dashed, solid, dotted and dashed curves correspond to $\left|\xi_{\mu \tau}\right|=5,10,20,30$, with $\delta_{\mu \tau}=0$. 

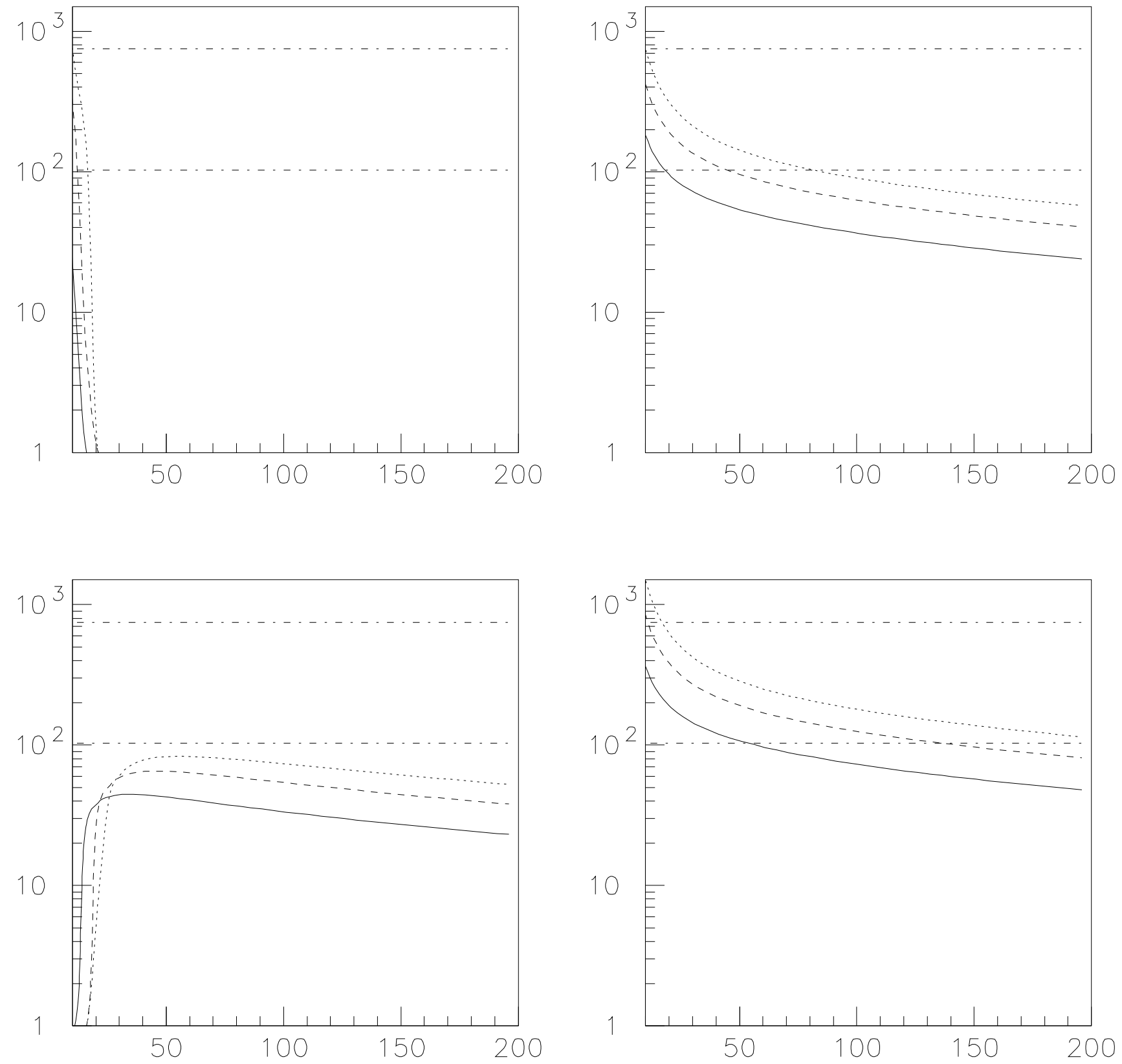